\section{EDITOR'S FOCUS}

\section{Volume 86 No. 6 December 2019}

\section{Early Career Investigator}

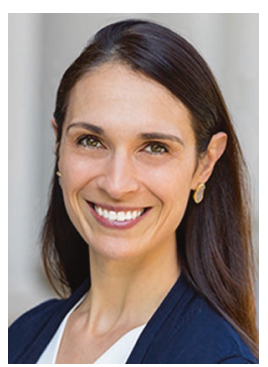

Congratulations to Anne Berens, the Early Career Investigator for December. A native of Massachusetts, Dr Berens studied first at Stanford, then in Rwanda, to pursue her interest in global health. Returning to Boston, she worked on a research program called Children and Early Adversity, followed by further public health training in London-all before medical school! As a medical student at Harvard, she developed an interest in how early developmental environments, including adverse exposures structured by social inequities, impact child health and well-being. She completed her pediatrics residency at the University of California at San Francisco and is preparing to start a fellowship next year in developmental-behavioral pediatrics at Stanford: a full circle. Her research, as reflected in Dr Berens and colleagues' article in this issue and the associated Comment, has policy implications. Her advice to those in early stages of academic medicine: a conventional path may not be the best for everyone; make your own choices regarding family, work, and service. See pages 682, 766 and 683

\section{Transformation of peripheral oxygen saturation to improve outcome predictability}

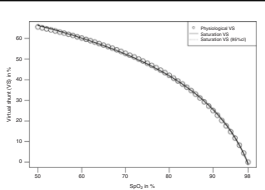

Zhou et al. used an empirically derived formula to transform peripheral oxygen saturation to a saturation virtual shunt. The shunt estimates linear disease severity and improves clinical prediction. See page 732
Modifiable pre-pregnancy and pregnancy antecedents associated with positive child health outcomes

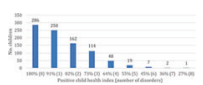

Using data from 889 children enrolled in the Extremely Low Gestational Age Newborn (ELGAN) study, Bangma et al. showed an association between higher scores on a positive child health index (PCHI) with medical interventions and higher socioeconomic status, and between lower PCHI scores with maternal chronic illness and high maternal body mass index. This article sparked two Comments on the definition of positive child health. Please join in the conversation! See pages 758, 690 and 688

Long-term and recent-onset obesity increases cardiometabolic risk in adolescence

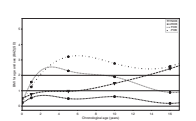

Obesity is a major public health concern worldwide, contributing to a decline in healthy years of life. The results of this large cohort study by Burrows et al. indicate that long-term and recent-onset obesity can equally increase cardiometabolic risk in adolescents. The findings point to a critical window of opportunity: tackling obesity during infancy, childhood, and adolescence might be an effective strategy for preventing persistence of obesity and reduce the risk of cardiometabolic diseases in adulthood. See page 776

\section{Cardiac tissue damage after cardiac arrest}

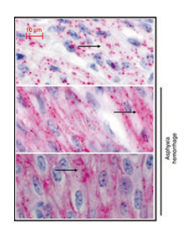

In this study by Weber et al., 44 neonatal piglets underwent asphyxia and hemorrhage, which led to cardiac arrest followed by resuscitation. Tissue was collected 4 hours after resuscitation. The results showed evidence of both structural and metabolic cardiac alterations, particularly connexin 43 translocation to cytosol, glycogen depletion, and local inflammation. See page 709

\section{Forskolin attenuates NLRP3 inflammasome activation}

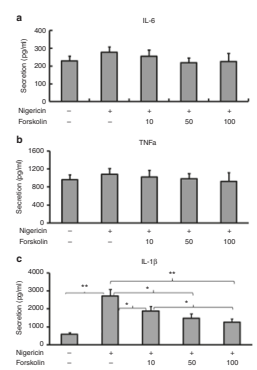

Chen et al. report that forskolin inhibits NLRP3 inflammasome activation and the secretion of mature interleukin-1 $\beta$ in human macrophages. Forskolin and other drugs that increase CAMP could represent a novel approach for treatment of pediatric diseases associated with excessive inflammasome activation. The Insights image in this issue is a professional illustration of the underlying mechanism. See pages 692 and 785

\section{Combined magnesium sulfate and hypothermia vs. hypothermia alone}

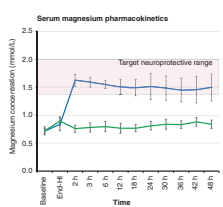

In a term neonatal encephalopathy model using piglets, Lingam et al. studied the neuroprotective efficacy of magnesium sulfate $\left(\mathrm{MgSO}_{4}\right)$ with hypothermia compared with hypothermia alone. $\mathrm{MgSO}_{4}$ bolus and infusion led to increased serum and cerebrospinal magnesium concentrations with decreased overall neuronal cell death but failed to show improvement in amplitude-integrated electroencephalogram score or magnetic resonance spectroscopy biomarkers. The findings provide highly valuable information given that the lack of benefit on validated measures of outcome suggests that a phase III clinical trial of add-on $\mathrm{MgSO}_{4}$ is unlikely to have a clinical impact. Galinsky et al. present a related editorial. See pages 699 and 685 